\title{
Changes in Brachial and Central Blood Pressure after Short Term Continuous Positive Airway Pressure Treatment of Patients with Moderate-to-Severe Obstructive Sleep Apnoea and Impaired Renal Function
}

\author{
Bodil G. Hornstrup ${ }^{*}{ }^{\oplus}$, Pia H. Gjørup ${ }^{2}$, Jost Wessels ${ }^{2}$, Thomas G. Lauridsen ${ }^{1,2}$, \\ Erling B. Pedersen', Jesper N. Bech1,2 (D) \\ ${ }^{1}$ University Clinic in Nephrology and Hypertension, Regional Hospital West Jutland and Aarhus University, Aarhus, Denmark \\ ${ }^{2}$ Department of Medicine, Regional Hospital West Jutland, Holstebro, Denmark \\ Email: *bodil.hornstrup@rm.dk
}

How to cite this paper: Hornstrup, B.G., Gjørup, P.H., Wessels, J., Lauridsen, T.G., Pedersen, E.B. and Bech, J.N. (2019) Changes in Brachial and Central Blood Pressure after Short Term Continuous Positive Airway Pressure Treatment of Patients with Moderate-to-Severe Obstructive Sleep Apnoea and Impaired Renal Function. Open Journal of Nephrology, 9, $1-19$.

https://doi.org/10.4236/ojneph.2019.91001

Received: December 12, 2018

Accepted: January 20, 2019

Published: January 23, 2019

Copyright ( 2019 by author(s) and Scientific Research Publishing Inc. This work is licensed under the Creative Commons Attribution International License (CC BY 4.0).

http://creativecommons.org/licenses/by/4.0/ (c) (i) Open Access

\begin{abstract}
Background: Previous studies of continuous positive airway pressure (CPAP) treatment for obstructive sleep apnoea (OSA) have shown conflicting results on the effect on blood pressure (BP), and patients with chronic kidney disease (CKD) have not been included in these studies. As OSA is a frequent comorbidity in patients with $\mathrm{CKD}$, it is of relevance to evaluate the effect of CPAP treatment on BP in this population. Aim: In this prospective follow-up study, we measured the effect of short term CPAP treatment of moderate-to-severe OSA on brachial and central BP, plasma level of syndecan-1 and vasoactive hormones, renal handling of sodium, subjective sleepiness, and quality of life in patients with impaired renal function. Methods: From December 2015 until March 2017, 25 patients were invited to participate in the study at the University Clinic in Nephrology and Hypertension, Aarhus University and Holstebro Hospital. At baseline and at follow-up after three to four months of CPAP treatment, we performed $24 \mathrm{~h}$ brachial and central ambulatory BP measurement, blood sampling measurements of plasma concentrations of syndecan-1, renin, angiotensin II, aldosterone, vasopressin, creatinine, haemoglobin A1c, and cholesterol, cardio respiratory monitoring, $24 \mathrm{~h}$ urine collection for measurement of urinary excretion of albumin, aquaporin-2, and epithelial sodium channel, Epworth Sleepiness Scale (ESS), and SF-36 (quality of life). Results: At follow-up, the 17 included patients with mean baseline estimated glomerular filtration rate $66 \mathrm{~mL} / \mathrm{min} / 1.73 \mathrm{~m}^{2}$ had a sig-
\end{abstract}


nificant decrease in systolic office-, $24 \mathrm{~h}$ - and daytime-BP $(13,7$, and $8 \mathrm{mmHg}$, respectively, $p<0.05)$, a non-significant reduction of nocturnal $\mathrm{BP}(6 \mathrm{mmHg})$. No changes was measured in frequency of non-dipping or in central $24 \mathrm{~h}$-, day- and nighttime-BP. Renal function remained unchanged, but urinary albumin excretion fell. ESS was unchanged. Quality of life improved. Conclusion: Short-term CPAP treatment of patients with moderate-to-severe OSA and reduced renal function decreased $24 \mathrm{~h}$ - and daytime-BP significantly and reduced urinary albumin excretion. Our results underline the importance of treatment of OSA in hypertensive patients with impaired renal function.

\section{Keywords}

Chronic Kidney Disease, Nocturnal Blood Pressure, Obstructive Sleep Apnoea, Central Blood Pressure, Continuous Positive Airway Pressure

\section{Introduction}

Obstructive sleep apnoea (OSA) is a frequent comorbidity in hypertension and chronic kidney disease (CKD) [1] [2]. OSA is characterised by obstructions of the upper airways during nighttime sleep causing repetitive pauses in breathings despite respiratory efforts. Termination of the obstructions requires arousal, which leads to poor sleep quality and daytime sleepiness in OSA patients. OSA patients show early signs of atherosclerosis and are of increased risk of stroke independent of other known risk factors such as hypertension, diabetes, smoking, and body mass index (BMI) [3] [4]. Furthermore, the nocturnal hypoxemia seen in OSA is associated to progression of renal failure [5] [6].

Continuous positive airway pressure (CPAP) has been an established treatment for OSA for many years with a documented improving effect on sleep, quality of life, and nocturnal hypoxemia [7] [8] [9]. High CPAP compliance is associated with lower risk of cardiovascular disease and with a renoprotective effect independent of blood pressure (BP) reduction [10] [11]. Different effects of CPAP treatment on BP levels have been reported; some studies show significant lowering effect [7], whereas other studies demonstrate sparse effect [12] [13] [14]. No studies have analysed the effect of CPAP treatment in patients with CKD; hence, short term effects (three to four months) of CPAP treatment of moderate-to-severe OSA in patients with $\mathrm{CKD}$ are unknown with respect to brachial BP, quality of life, and sleep symptoms.

Central aortic systolic pressure (CASP) may provide additional information on the level of arteriosclerosis and cardiovascular disease associated end organ damage [15] [16]. CPAP treatment has been shown to lower the central systolic pressure using twice-a-day measurements in normo- and uncomplicated essential hypertensive patient with OSA [17]. However, the effect of CPAP treatment on CASP has not been clarified in CKD patients.

Increased syndecan-1 in plasma is an indication of increased shedding from 
the glycocalyx protection layer as a response to cardiovascular stress [18]. We have previously found increased syndecan-1 levels in hypertensive patients compared with healthy controls [19]. However, it is not known, whether CPAP treatment can alter plasma levels of syndecan-1, and thereby be an indicator of reduced shedding as a response to CPAP treatment.

The aim of this intervention study was to evaluate the effect of short term CPAP treatment in subjects with moderate-to-severe OSA (apnoea hypopnoea index (AHI) >15) and impaired renal function (estimated glomerular filtration rate (eGFR) $15-89 \mathrm{~mL} / \mathrm{min} / 1.73 \mathrm{~m}^{2}$ at sampling time) on 1) brachial and central BP, 2) p-syndecan-1,3) plasma levels of renin, angiotensin II, aldosterone, and vasopressin, 4) eGFR and urinary excretion of albumin, aquaporin-2, and a fraction of the epithelial sodium channel, 5) quality of life, and 6) subjective sleepiness.

\section{Materials and Methods}

\subsection{Design}

The project was carried out as a prospective intervention study. Patients were included at the time of start of CPAP treatment and followed up after approximately three to four month.

\subsection{Study Settings}

The study was conducted at the University Clinic in Nephrology and Hypertension, Aarhus University and Holstebro Hospital, and the Sleep Apnoea Clinic, Department of Medicine, Holstebro Hospital. The recruitment period was from December 2015 until March 2017.

\subsection{Patients}

Patients were recruited from the Renal Outpatient Clinic, Holstebro Hospital (eGFR $15-59 \mathrm{~mL} / \mathrm{min} / 1.73 \mathrm{~m}^{2}$ ) or from a population study in Holstebro County (diagnosis of hypertension and eGFR $60-89 \mathrm{~mL} / \mathrm{min} / 1.73 \mathrm{~m}^{2}$ ) [20]. All patients were diagnosed with moderate-to-severe OSA and had not received CPAP treatment previously. The patients were examined for OSA during participation in two other projects by the same authors [19] [21]. They were offered CPAP treatment according to usual clinical practice.

Inclusion criteria: men and women, age 18 - 80, eGFR 15 to $89 \mathrm{~mL} / \mathrm{min} / 1.73$ $\mathrm{m}^{2}$, OSA with apnoea hypopnoea index (AHI) $\geq 15$. Exclusion criteria: unwillingness to participate, malignant disease, drug abuse, alcohol abuse $(>21 />14$ drinks per week for males and females, respectively), atrial fibrillation or heart failure, liver disease (alanine aminotransferase $>200 \mathrm{U} / \mathrm{L}$ ), severe chronic obstructive lung disease (forced expiratory volume in 1 second $\left(\mathrm{FEV}_{1}\right)$ less than $50 \%$ of expected), and difference in BP between right and left arm above 10/10 $\mathrm{mmHg}$. Withdrawal criteria: development of exclusion criteria, lack of completion of participation, and lack of compliance. 
Number of subjects: the minimal relevant difference in mean $24 \mathrm{~h}$ systolic BP (SBP) was $10 \mathrm{mmHg}$ with standard deviation (SD) 10 . With a statistical power of $80 \%$ and a significance level of $5 \%$, it was calculated that the number of subjects should be at least 16 .

\subsection{Ethics}

This study was reviewed and approved by the Central Denmark Region Committees on Health Research Ethics (j.no.: M-2013-285-13 and M-2013-304-13) and by the Danish Data Protection Agency (j.no.: 1-16-02-399-13 and 1-16-02-458-13). The study was carried out in accordance with the Helsinki Declaration. All study patients received oral and written information about the project and provided informed written consent prior to study enrolment. ClinicalTrials.gov registration identification was NCT01951248 and NCT02078778.

\subsection{End Points}

The primary end point was change in brachial $24 \mathrm{~h}$ SBP after three to four months of CPAP treatment.

The secondary end points were changes in: $24 \mathrm{~h}$ central BP, relative nocturnal brachial SBP and CASP decrease, urinary excretion rate of aquaporin-2 (u-AQP2) and epithelial sodium channel fraction $\gamma(\mathrm{u}-\mathrm{ENaC} \gamma), 24 \mathrm{~h}$ urine excretion of albumin (u-albumin), p-syncecan-1, vasoactive hormones (plasma renin concentration, plasma aldosterone, plasma angiotensin II, plasma arginine vasopressin (p-AVP)), reporting of subjective sleepiness (Epworth Sleepiness Scale, ESS), and quality of life (by SF-36 questionnaire).

\subsection{Blood Pressure}

Twenty-four hour ambulatory BP measurement (ABPM) was carried out using an oscillometric device, A\&D TM-2430 (A\&D Company Limited, Tokyo, Japan). An appropriate size cuff was chosen after measuring the upper arms circumference and placed on the right side. Twenty-four hour CASP was measured using applanation tonometry by BPro Health Stat (BPro, HealthSTATS, Singapore). The BPro device was placed on the left wrist after being calibrated with a mean of the last three of four consecutive BP measurements on the left arm derived from the A\&D device used for $24 \mathrm{~h}$ brachial ABPM on the same subject.

$\mathrm{BP}$ was measured every $15 \mathrm{~min}$ and every $30 \mathrm{~min}$ during day- and nighttime, respectively, by the A\&D device, and every 15 min by the BPro device throughout the 24 hours. ABPM were considered satisfactory with 14 or more daytime recordings (fixed daytime setting at $6 \mathrm{am}$ to $11 \mathrm{pm}$ ) and seven or more nighttime recordings (fixed nighttime setting at $11 \mathrm{pm}$ to $6 \mathrm{am}$ ).

A semiautomatic oscillometric device, Omron 705IT (Omron Matsusaka CO, Ltd., Matsusaka City, Japan) was used for bilateral brachial BP measurements with the subject sitting in upright position after minimum ten minutes rest.

Hypertension was defined as brachial $24 \mathrm{~h} \mathrm{BP} \geq 130 \mathrm{mmHg}$ systolic and/or $\geq$ 
$80 \mathrm{mmHg}$ diastolic, non-dipping was defined as relative nocturnal systolic BP decrease $\leq 10 \%$, and resistant hypertension was defined as hypertension on three antihypertensive drugs, one of these being a diuretic. These definitions are according to most recent guidelines from the European Society of Hypertension/ European Society of Cardiology [22].

\subsection{Sleep Apnoea}

An ambulatory cardio respiratory monitoring (CRM) was performed using Embletta Gold (Natus Medical Incorporated, USA) in all participants at baseline and follow-up. RemLogic-E Software was used for analysing and storing data. The sleep report was generated from sleep time and consisted of continuous recordings from the Embletta monitor. During sleep, this monitor recorded air flow from a nasal pressure transducer, arterial oxygen saturation from a pulse oximeter, respiratory effort from thoracic and abdominal impedance belts, and body position from sensors. An apnoeic event was defined as a cessation of nasal airflow accompanied by a drop of the signal below $10 \%$ of the reference amplitude for an interval of 10 seconds. A hypopnoeic event was defined as a reduction of the signal below $70 \%$ of the reference amplitude for an interval of 10 seconds with a subsequent desaturation event no later than 20 seconds after the start of the event. An oxygen desaturation event was observed by a decrease in oxygen saturation by at least $4 \%$. Events lasting longer than 120 seconds (apnoea, hypopnea, or desaturation) were excluded.

Apnoea hypopnoea index (AHI) was defined as the sum of apnoeas and hypopnoeas per hour of registered sleep. Oxygen desaturation index (ODI) was defined as oxygen desaturation events pr. hour of sleep. OSA was defined as AHI $\geq$ 5 , moderate-to-severe OSA $\geq 15$. Definitions above are according to recommendations from the American Academy of Sleep Medicine (AASM) [23].

\subsection{Continuous Positive Airway Pressure (CPAP)}

OSA was treated with AirSense 10 AutoSet (ResMed/Maribo Medico) or REMstar Auto with A-Flex (Phillips/Respironics). Treatment was started and followed up by trained nurses in the Sleep Apnoea Clinic, Department of Medicine, Holstebro Hospital, according to standard procedure. A mask for the CPAP treatment was chosen to the best fit for the patient, either nasal or full face mask. The two systems had identical functionality and were self-adjusting; the pressure was automatically adjusted in response to inhalation flow, snoring, and apnoeas. The subjects were instructed in correct use of the system, change of air filter, and daily maintenance/cleaning of the system. Humidifier was added to the system if needed. Adherence was defined from the last month of treatment as percentage of 4 hours every night; the patient had been using the equipment.

\subsection{Experimental Procedures}

Subjects received oral and written information about this project after being diagnosed with moderate-to-severe OSA $(\mathrm{AHI} \geq 15)$ in previous studies. An in- 
formation meeting was set up, and after informed written consent, life style questionnaire (SF-36) was filled in by the patient alone after instructions were given, and follow-up meeting was planned. Baseline information on ABPM, CRM, blood and urine samples, medical treatment, sleep symptoms (Epworth Sleepiness Scale (ESS)), and medical history were collected from the previous project participation.

While participating in this study, patients followed their usual consultations in the Renal Outpatient Clinic or general practitioners with regard to their renal disease or hypertensive disease. At these consultations, antihypertensive medications were adjusted if necessary. This was assessed by the patient's physician in the Outpatient Clinic or the general practitioner.

At baseline and at follow-up after approximately three to four months of CPAP treatment, subjects were examined as described in the following section. Blood samples were drawn after $20 \mathrm{~min}$ of rest in supine position. Twenty-four hour brachial and central ABPM were carried-out simultaneously, and $24 \mathrm{~h}$ urine collection was performed and returned at the latest 4 hour after completion. CRM, as described above, was completed, and at follow-up the CRM was carried-out simultaneously with CPAP treatment, and adherence was registered from the CPAP device. Information on changes in use of medication and eventual new medical events were obtained by the electronic patient record and questionnaire, respectively. Current quality of life and subjective sleepiness were reported by SF-36 and ESS, respectively. These questionnaires were filled in by the participant alone after a short introduction.

\subsection{Biochemical Analyses}

Blood samples were centrifuged at $4^{\circ} \mathrm{C}$ for $10 \mathrm{~min}$ at $2200 \times$ g. Plasma samples were kept frozen at $-20^{\circ} \mathrm{C}$ (P-Angiotensin II) and $-80^{\circ} \mathrm{C}$ (Syndecan, plasma renin concentration, $\mathrm{P}$-aldosterone, and $\mathrm{p}$-AVP), and urine samples at $-20^{\circ} \mathrm{C}$ until assayed.

Urinary and plasma osmolality were measured by freezing-point depression (Advanced Model 3900 multisampling osmometer).

Plasma renin concentration was determined by radioimmunoassay using a kit from CIS Bio International, Gif-Sur-Yvette Cedex, France. Minimal detection level was $1 \mathrm{pg} / \mathrm{mL}$. The coefficients of variations were $14.5 \%$ (inter-assay) and $4.5 \%$ (intra-assay).

$\mathrm{P}$-angiotensin II and p-AVP were extracted from plasma with C18 Sep-Pak (Water associates, Milford, MA, USA), and subsequently determined by radioimmunoassay [24] [25]. The antibody against Ang II was obtained from the Department of Clinical Physiology, Glostrup Hospital, Denmark. Minimal detection level was $2 \mathrm{pmol} / \mathrm{L}$. The coefficients of variation were $12 \%$ (inter-assay) and 8\% (intra-assay). The antibody against AVP was a gift from Professor Jacques Dürr, Miami, FL., USA. The coefficients of variation were $13 \%$ (inter-assay) and 9\% (intra-assay). Minimal detection level was $0.5 \mathrm{pmol} / \mathrm{L}$.

P-aldosterone was determined by radioimmunoassay using a kit from 
Demeditec Diagnostics GmbH, Kiel, Germany. The coefficients of variations were $17.2 \%$ (inter-assay) and $12.6 \%$ (intra-assay). Minimal detection level was $3.99 \mathrm{pmol} / \mathrm{L}$.

P-syncecan-1 was analysed on EDTA-plasma using a human ELISA kit from Abcam plc, Cambridge, United Kingdom. The coefficients of variations were $10.2 \%$ (inter-assay) and 6.2\% (intra-assay). Minimal detection level was $4.94 \mathrm{ng} / \mathrm{mL}$.

U-AQP2 was determined by RIA as previously described [26] [27]. Rabbit anti-AQP2 antibodies were a gift from Professor Soren Nielsen and Professor Robert Fenton, the Water and Salt Research Center, Aarhus University, Denmark. Coefficients of variation: $11.7 \%$ (inter-assay) and 5.9\% (intra-assay). Minimal detection level: $32 \mathrm{pg} / \mathrm{tube}$.

U-ENaC $\gamma$ was measured by RIA as described previously [28] [29]. ENaC $\gamma$ was synthesized and purchased by Lofstrand, Gaithersburg, Maryland, USA. The ENaC $\gamma$ antibody was a gift from Professor Soren Nielsen and Professor Robert Fenton, the Water and Salt Center, Aarhus University. Coefficients of variation: $10 \%$ at a mean level of $338 \mathrm{pg} /$ tube (inter-assay), $9 \%$ at a mean level of $743 \mathrm{pg} /$ tube (inter-assay), 5.0\% in the range 125 - $135 \mathrm{pg} /$ tube (intra-assay), and 5.6\% in the range 290 - 380 pg/tube (intra-assay). Minimal detection level: 35 pg/tube.

Plasma levels of creatinine, haemoglobin A1c (HbA1c), cholesterols, and urinary concentrations of albumin, creatinine, and sodium were measured using routine methods at the Department of Clinical Biochemistry, Holstebro Hospital, Denmark. eGFR was calculated by the MDRD-equation.

\subsection{Statistical Methods}

Statistical analyses were performed by the authors using IBM SPSS statistics version 22 (IBM Corp.; Armonk, NY, United States). All data were tested for normality and variance equality. The statistical level of significance was $p<0.05$ in all analyses.

Continuous variables were reported as means with SD or as median with interquartile rang $[25 ; 75]$ depending on whether the data were normally distributed or not. Categorical variables were reported as percentages with number. Paired t-tests were used for paired continuous variables with normally distributed differences; otherwise Wilcoxon signed rank test was used. McNemars test was used for paired categorical data. Univariate analyses were performed using Pearson's test or Spearman Rho test on normally distributed or non-normally distributed continuous variables, respectively.

The SF36 questionnaire filled out by the patients was scored using the procedure from International Resource Center for Health Care Assessment (Boston, MA), called RAND 36-Item Health Survey 1.0 [30].

\section{Results}

\subsection{Demographics}

Twenty-five patients were invited to participate; two were not included as they never began CPAP treatment despite initial intention to do so. Six were ex- 
cluded; four due to early discontinuation of treatment (within one month), and two due to uncompleted follow-up examination (incomplete data). Seventeen participants completed follow-up examination (Figure 1).

Baseline clinical, laboratory, and sleep characteristics of the included $17 \mathrm{pa}-$ tients are presented in Table 1. Mean eGFR were $66 \mathrm{~mL} / \mathrm{min} / 1.73 \mathrm{~m}^{2}$, and 36\% $(n=6)$ were diagnosed with diabetes. All participants were diagnosed with hypertension, and $76 \%(\mathrm{n}=13)$ received antihypertensive medication. Of them, $23 \%(n=3)$ were controlled hypertensive, and $54 \%(n=7)$ had resistant hypertension.

At baseline and follow-up, subjects received a mean of $3.7 \pm 4.4$ and $3.8 \pm 4.5$ defined daily doses (DDD) of BP lowering drugs, respectively $(p>0.05)$. Twelve subjects' DDD remained unchanged, one subject's DDD decreased (0.25 DDD), and four subjects' DDD increased (mean change $0.8 \mathrm{DDD}$ ). Forty-two percent $(n=7)$ received statins throughout the follow-up period.

\subsection{Brachial Blood Pressure}

Table 2 shows baseline and follow-up brachial BP; brachial systolic and diastolic office, $24 \mathrm{~h}$ and daytime BP decreased significant at follow-up. No changes were observed in nocturnal BP or nocturnal BP decrease. The frequency of non-dipping was unchanged (at baseline $47 \%(\mathrm{n}=8)$ and follow-up 53\% $(\mathrm{n}=9)$, $p=1.0($ Figure $2(\mathrm{a}))$.

\subsection{Central Arterial Systolic Pressure}

Two patients' baseline and one patient's follow-up CASP measurement was excluded from analysis due to few measurements Therefore, CASP analyses included 14 patients' measurements. There was a non-significant decrease in $24 \mathrm{~h}$, day and nighttime CASP (Table 2). Non-dipping was seen in $64 \%(\mathrm{n}=9)$ at baseline, and $86 \%(\mathrm{n}=12)$ at follow-up $(p=0.45)$ (Figure 2(b)).

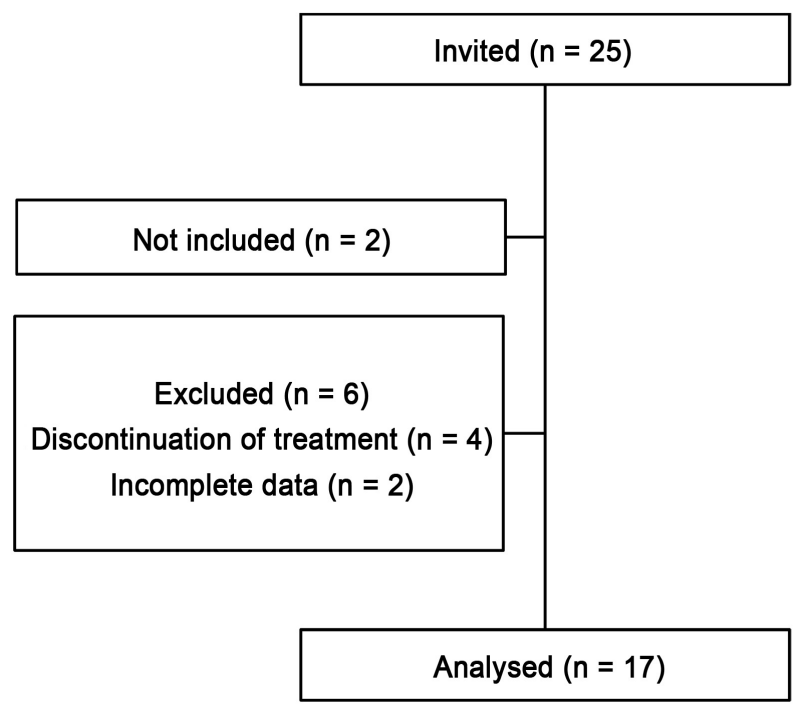

Figure 1. Flow chart of patients. 


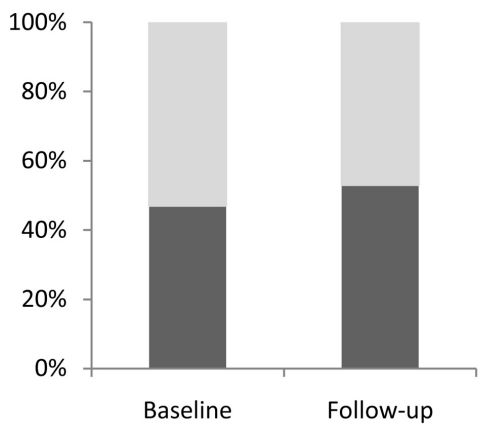

(a) Brachial

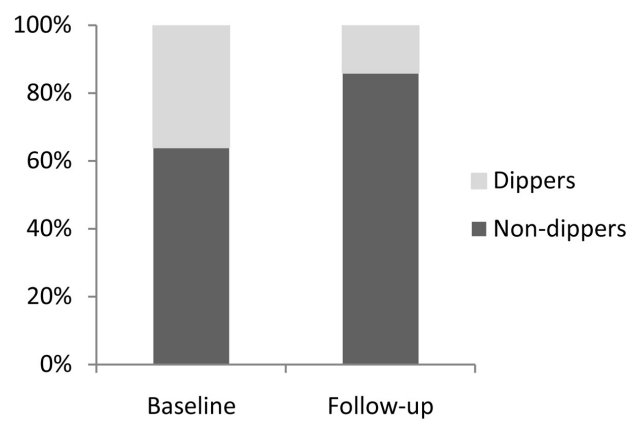

(b) CASP

Figure 2. Percentage of patients with dipping/non-dipping blood pressure pattern at baseline and follow-up in brachial (a) and CASP (b) values. Percentage (number) of subjects with nocturnal systolic blood pressure decrease $</ \geq 10 \%$ of day time blood pressure as non-dippers/dippers, respectively. Abbreviations. CASP: central aortic systolic pressure. Brachial: $p=1.0$, CASP: $p=0.45$. Statistics were performed using McNemars test.

Table 1. Demographics and sleep characteristics at baseline.

\begin{tabular}{cc}
\hline Demographic Characteristics & $\mathbf{N}=17$ \\
Age, years & $65(7)$ \\
Gender, male, $\%(\mathrm{n})$ & $77(13)$ \\
Body mass index, $\mathrm{kg} / \mathrm{m}^{2}$ & $34(7)$ \\
Estimated glomerular filtration rate, $\mathrm{ml} / \mathrm{min} / 1.73 \mathrm{~m}^{2}$ & $66(23)$ \\
U-albumin, $\mathrm{mg} / 24 \mathrm{hour}$ & $11[7 ; 138]$ \\
Haemoglobin Alc, mmol/mol & $49(15)$ \\
Total cholesterol, mmol/L & $4.9(1.0)$ \\
High density lipoprotein cholesterol, mmol/L & $1.1(0.3)$ \\
Triglyceride, mmol/L & $2.2(1.1)$ \\
\hline Sleep Characteristics & $5.0[3.5 ; 8.5]$ \\
\hline Epworth Sleepiness Scale (ESS), 0 - 24 $4^{\mathrm{a}}$ & $33[20 ; 37]$ \\
Apnoea hypopnoea index (AHI), events pr. hour & $36[17 ; 51]$ \\
Oxygen desaturation index (ODI), events/hour & $92(2)$ \\
Mean oxygen saturation, \% &
\end{tabular}

Baseline demographic characteristics and baseline sleep data from all 17 patients. Data are presented as mean (SD) except u-albumin, Epworth Sleepiness Scale, Apnoea hypopnoea index, and oxygen desaturation

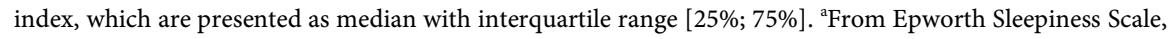
self-reported data.

The changes in brachial and central BP from baseline to follow-up were similar (data not shown).

\subsection{Sleep Examination}

Follow-up time was median 89 days with a total range from 72 to 139 days. Data from sleep examination at baseline are showed in Table 1. At follow-up, AHI and ODI decreased and mean oxygen saturation $\left(\mathrm{SaO}_{2}\right)$ increased, while sleep symptoms (ESS) were reported unchanged (Table 3). Adherence to CPAP 
Table 2. Brachial blood pressure and central aortic systolic pressure at baseline and at follow-up.

\begin{tabular}{ccccc}
\hline & \multicolumn{2}{c}{ Brachial $(\mathrm{n}=17)$} & \multicolumn{2}{c}{ CASP ( $=14)$} \\
\cline { 2 - 5 } & Baseline & Follow-up & Baseline & Follow-up \\
\hline Office BP systolic, mmHg & $149(13)$ & $136(15)^{*}$ & & \\
Office BP diastolic, mmHg & $87(7)$ & $79(12)^{*}$ & & \\
24 hour BP, systolic, mmHg & $143(11)$ & $136(8)^{*}$ & $125(16)$ & $117(16)$ \\
24 hour BP, diastolic, mmHg & $81(7)$ & $78(7)^{* *}$ & & \\
24 hour heart rate, beats/min & $68(7)$ & $68(7)$ & & \\
Daytime BP, systolic, mmHg & $146(11)$ & $138(9)^{*}$ & $127(16)$ & $118(16)$ \\
Daytime BP, diastolic, mmHg & $83(8)$ & $79(7)^{*}$ & & \\
Nighttime BP, systolic, mmHg & $131(16)$ & $125(14)$ & $121(17)$ & $112(16)$ \\
Nighttime BP, diastolic, mmHg & $74(6)$ & $72(7)$ & & \\
Absolute nocturnal BP decrease, mmHg & $15(12)$ & $13(14)$ & $7(9)$ & $7(8)$ \\
Relative nocturnal BP decrease, \% ${ }^{\mathrm{a}}$ & $10(8)$ & $9(10)$ & $5(7)$ & $6(7)$ \\
\hline
\end{tabular}

Abbreviations: BP: blood pressure. CASP: central aortic systolic pressure. Office blood pressure obtained from baseline and follow-up examinations. $24 \mathrm{~h}$, day and nighttime brachial and CASP data obtained from $24 \mathrm{~h}$ ABPM. CASP measurements only consist of systolic values from $24 \mathrm{~h}$ central ABPM. Data are pre-

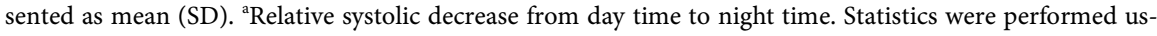
ing paired t-test. ${ }^{*} p<0.05,{ }^{* *} p<0.001$.

Table 3. Changes in body mass index, laboratory results, and sleep parameters at follow-up.

\begin{tabular}{ccc}
\hline $\mathbf{n}=\mathbf{1 7}$ & $\boldsymbol{\Delta}$ value & $\boldsymbol{p}$ \\
\hline Metabolic parameters & & \\
\hline Body mass index, $\mathrm{kg} / \mathrm{m}^{2}$ & $0.2(4.8)$ & 0.88 \\
Haemoglobin A1c, $\mathrm{mmol} / \mathrm{mol}$ & $0.7(4.4)$ & 0.55 \\
Total cholesterol, mmol/L & $-0.3(1.1)$ & 0.25 \\
High density lipoprotein cholesterol, mmol/L & $-0.0(0.2)$ & 0.67 \\
Triglyceride, mmol/L & $-0.2(0.9)$ & 0.31 \\
\hline Renal parameters & $0.7(10.4)$ & 0.77 \\
\hline U-albumin, mg/24 hour & $-3[-1 ; 31]$ & 0.025 \\
\hline Sleep parameters & $-1[-3 ; 1]$ & 0.112 \\
\hline Eptimated glomerular filtration rate, $\mathrm{mL} / \mathrm{min} / 1.73 \mathrm{~m}^{2}$ & $-27[-19 ;-38]$ & $<0.0001$ \\
Apnoea hypopnoea index (AHI), events pr. hour & $-33[-15 ;-48]$ & $<0.0001$ \\
Oxygen desaturation index (ODI), events/hour & $3(2)$ & 0.001 \\
\hline Mean oxygen saturation, $\%$ & & \\
\hline
\end{tabular}

Values represent follow-up minus baseline values. U-albumin, Epworth Sleepiness Scale, Apnoea hypopnoea index, and Oxygen desaturation index are presented as median with interquartile range [25\%; 75\%]. The remaining data are presented as mean (SD). ${ }^{\mathrm{a}}$ From Epworth Sleepiness Scale, self-reported data. Statistics were performed using paired t-test or Wilcoxon signed rank test. 
treatment was median $73 \%$ [57; 93], total range from $20 \%$ to $100 \%$. There was no correlation between changes in BP parameters and adherence or follow-up time (data not shown). When excluding subjects with CPAP adherence below 50\% $(\mathrm{n}=3)$, BP changes on all parameters were the same as for the whole group (data not shown).

Univariate correlation analysis showed no association between baseline AHI and changes in BP. Baseline AHI was correlated to changes in ESS ( $r=0.62, p=$ 0.008), but not to baseline ESS or adherence to CPAP treatment.

\subsection{Renal and Metabolic Parameters}

Changes in metabolic and renal characteristics at follow-up are shown in Table 3; no significant changes were observed in BMI, HbA1c, or cholesterols. Renal function remained unchanged, whereas albuminuria decreased significantly 3 $[-1 ; 31] \mathrm{mg} / 24 \mathrm{~h}(p=0.025)$. There was no association between changes in BP and eGFR at baseline or at follow-up, and the reduction in albuminuria was not related to changes in any of the BP parameters, eGFR (at baseline or at follow-up), or mean $\mathrm{SaO}_{2}$ at follow-up (tested by univariate correlation analysis, data not shown).

\subsection{SF36-Questionnaire on Quality of Life}

Patients experienced improved quality of life with regard to vitality, social functioning, emotional role, and mental health, whereas no significant changes in physical functioning, physical role, general health, or bodily pain were seen (Table 4). Baseline eGFR was significantly associated to follow-up SF-36 score ( $\mathrm{r}$ $=0.50, p=0.042)$, but not baseline SF-36 score $(\mathrm{r}=0.48, p=0.052)$. No correlation of SF-36 baseline or follow-up and AHI or Epworth at baseline or follow-up.

Table 4. SF36 scores at baseline and follow-up.

\begin{tabular}{cccc}
\hline & Baseline & Follow-up & $p$ \\
\hline Physical Functioning & $78(31)$ & $79(32)$ & 0.78 \\
Physical Role & $63(49)$ & $67(48)$ & 0.42 \\
General Health & $67(26)$ & $69(26)$ & 0.55 \\
Vitality & $65(26)$ & $73(23)$ & 0.0004 \\
Bodily Pain & $73(17)$ & $77(23)$ & 0.37 \\
Social Functioning & $87(19)$ & $94(11)$ & 0.008 \\
Emotional Role & $69(48)$ & $78(42)$ & 0.02 \\
Mental Health & $86(20)$ & $91(15)$ & 0.006
\end{tabular}

Scores for quality of life using SF36 questionnaire at baseline and at follow up from all 17 participants. Data are presented as mean (SD). Procedure for scoring the SF36 questionnaire was RAND 36-Item Health Survey 1.0 from International Resource Center for Health Care Assessment (Boston, MA). The scales range from 0 (minimal well-being) to 100 (maximal well-being). Statistics were performed using paired t-test. 


\subsection{Plasma Levels of Syndecan-1}

There was no change in p-syncecan-1 from baseline to follow-up $(18[15 ; 25]$ vs. $19[16 ; 23] \mathrm{ng} / \mathrm{mL}, p=0.41)$.

\subsection{Plasma Levels of Vasoactive Hormones}

No changes were seen from baseline to follow-up in plasma levels of the vasoactive hormones; P-aldosterone $(159[131 ; 259]$ vs. $183[115 ; 268] \mathrm{pmol} / \mathrm{L}, p=$ $0.87), \mathrm{P}$-angiotensin II $(13[7 ; 55]$ vs. $11[7 ; 30] \mathrm{pg} / \mathrm{mL}, p=0.11)$, plasma renin concentration $(26[8 ; 67]$ vs. $16[10 ; 48] \mathrm{pg} / \mathrm{mL}, p=0.83)$, or AVP $(0.4[0.4 ; 0.7]$ vs. $0.5[0.3 ; 0.8] \mathrm{pmol} / \mathrm{L}, p=0.28)$.

\subsection{Urinary Excretion of $\mathrm{AQP} 2$ and $\mathrm{ENaCy}$}

From baseline to follow-up, no significant changes were seen in urinary excretion of AQP2 $(1.4[1.1 ; 1.8]$ vs. $1.3[1.1 ; 2.6] \mathrm{ng} / \mathrm{min}, p=0.88)$ or $\mathrm{ENaC} \gamma(1.2$ $[1.0 ; 1.6]$ vs. $1.2[0.9 ; 1.8] \mathrm{ng} / \mathrm{min}, p=0.54)$.

\section{Discussion}

The main findings of this study were a statistically significant and clinically relevant decrease in $24 \mathrm{~h}$ and daytime $\mathrm{BP}$ after short term CPAP treatment in patients with impaired renal function (mean eGFR $66 \mathrm{~mL} / \mathrm{min} / 1.73 \mathrm{~m}^{2}$ ) and a reduction in albuminuria. The decrease in nocturnal BP was not statistically significant, and there was no difference in nocturnal BP decrease or frequency of non-dipping. Renal function remained unchanged.

Previous studies reported different results of the BP response to CPAP. Becker et al. found a significant ( $>10 \mathrm{mmHg}$ decrease) in systolic and diastolic using 24 hours BP measuring, both during day- and nighttime [31], Hermida et al. found a small $(<3 \mathrm{mmHg})$ and statistically non-significant decrease in $24 \mathrm{~h} \mathrm{BP}$ [32], whereas Durán-Cantolla et al. [33] measured a similar and significant fall in $24 \mathrm{~h}$, day and nighttime BP. In the latter study, the number of non-dippers was reduced after treatment [33]. All studies comprised mainly hypertensive patients diagnosed with OSA, whereas no information was given about the patients' renal function and eventually diabetes. Hence, differences in baseline characteristics between studies may explain some of the divergent findings of BP response. Moreover, patients in the studies had different baseline levels of BP, AHI, and ESS, which may also influence the response to CPAP.

In the present study, five patients had changed their antihypertensive treatment. However, the difference in mean DDD at baseline and follow-up was less than $3 \%$. We do not believe that such a small change in DDD can solely explain the $\mathrm{BP}$ response at follow-up.

Our study, in contrast to the previous studies, included patients with known impaired renal function. Patients were recruited from a renal outpatient clinic, and not primarily based on referral to a sleep clinic, which most likely explains why the patients in the present study had lower AHI and less sleep symptoms 
compared with previous studies. However, in the present population, we demonstrated that it is possible to lower BP in patients with moderate-to-severe OSA using CPAP treatment. A previous meta-analysis of CKD patients demonstrated that a $5 \mathrm{~mm} \mathrm{Hg}$ reduction in office BP reduced the risk of major cardiovascular events with $17 \%$. Thus, the reduction we demonstrated in this population is of clinical relevance. One prevalence study found that as many as $40 \%$ of CKD3-4 patients suffered from moderate-to-severe OSA [1], whereas we in a preceding study only found moderate-to-severe OSA present in $22 \%$ of CKD3-4 patients [21]. However, based on our studies, we find it reasonable to suggest that patients with CKD should be examined for presence of OSA and treated, if moderate-to-severe OSA is present.

We also investigated the effects of CPAP treatment on central blood pressure derived from applanation tonometry. To our knowledge, no data have been reported on $24 \mathrm{~h}$ ambulatory central BP changes as response to CPAP treatment. Prior to our study, Litvin et al. [34] and Hoyos et al. [35] reported a decrease in central BP measured as spot measurements in an in-office set-up after three and eight weeks CPAP treatment, respectively. Both studies found a similar decrease in $\mathrm{mmHg}$ in central and peripheral BP after treatment. In agreement with the previous studies, we found a tendency to a decrease in central BP, however, in our study not statistically significant.

As expected, we found that AHI and ODI decreased, and mean oxygen saturation increased at follow-up in good agreement with a Cochrane review [7]. These findings also documented that patients adhered to the treatment.

In our study we found a decreased level of urinary excretion of albumine at follow-up, and renal function remained unchanged measured by eGFR. We did not demonstrate any correlation between the BP changes and renal function. We did not demonstrate any changes in metabolic parameters (blood cholesterols, HbA1c, or BMI). Previous studies have evaluated the effect of CPAP treatment on renal parameters. In a retrospective cohort study, Puckrin et al. reported slower progression of renal disease and lower levels of proteinuria in CKD3-5 patients with adherence to CPAP than non-adherent counterparts [10], but BP levels were unchanged at follow-up. Thus, presumably, BP is not, or at least not the only, important factor. Other authors have shown an association between nocturnal hypoxia and declining renal function [5] [6]. Hence, elevated oxygen levels associated to CPAP treatment may be of importance in explaining the possible renoprotective effect of CPAP treatment on renal function. However, we did not demonstrate this possible association in our study. However, the finding of reduced albuminuria after CPAP treatment adds to the beneficial profile of this treatment in patients with CKD and OSA. As in previous studies, we did not demonstrate any effect of CPAP treatment on BMI or other related metabolic factors [36] [37]. However, even though patients were obese, follow-up time was relatively short to expect changes in BMI and the other parameters without any life style intervention. 
In the present study, we did not demonstrate any significant change in reporting of sleep symptoms (ESS). Self-reported quality of life (SF-36) was reported higher on mental parameters at follow-up. Other studies have demonstrated less pronounced sleep symptoms after CPAP treatment [7] [37] [38]. These studies comprised subjects with both higher and identical levels of sleep symptoms as in our study. However, none of the participants in these studies suffered from CKD. Hence, in our population, sleep symptoms may be more related to renal disease than OSA, blunting the effect of CPAP treatment. The changes in ESS were correlated to AHI. This implies that the effect on sleep symptoms is more well-defined in more severe degrees of OSA.

Although patients in the present study suffered from renal failure and had hypertension and diabetes as frequent comorbidities, we demonstrated improved quality of life with respect to vitality (energy and fatigue) and mental parameters with no changes in physical parameters. Two previous studies have reported similar or more pronounced improvements of quality of life after $4-6$ weeks CPAP treatment [8] [39]. In one of these studies, participant suffered from more severe OSA, and in both, patients reported more severe sleep symptoms. Interestingly, we observed an improvement of quality of life in a heavier diseased population with less severe OSA and sleep symptoms. However, eGFR was correlated to overall quality of life at follow-up, which suggest that presence of renal disease is an important factor for quality of life in our population. This may be one explanation for the lack of effect on the patient's physical health parameters in this study.

We did not demonstrate any changes in p-syndecan-1. In vascular diseases, syndecans are shedded from the glycocalyx protection layer in the cardiovascular system [18]. In a previous study, we demonstrated higher plasma levels of syncecan-1 in a hypertensive population with sparse disease burden compared to healthy controls [19]. Other studies have reported higher levels of syndecans associated to declining renal function in a CKD population [40] and to overt heart failure in hypertensive patients [41]. We did not demonstrate any changes in syncecan-1 levels after CPAP treatment. We included only a small population with other cardiovascular risk factors. The over-all cardiovascular risk profile may be a stronger negative influence than an eventual positive influence of CPAP treatment.

We did not demonstrate any effect on renal handling of sodium $(\mathrm{ENaC} \gamma$ and $\mathrm{AQP} 2$ ) or vasoactive hormones in relation to CPAP treatment. One of the explanations for the high occurrence of OSA in CKD patients is sodium and water retention mediated by abnormal renin-angiotensin-aldosterone system (RAAS) activity [42]. In patients with OSA, an abnormal diurnal RAAS activity has been reported [43], and one study has shown, that both OSA severity and BP levels were reduced when adding aldosterone antagonists to the antihypertensive treatment in hypertensive OSA patients [44]. Hence, when analysing the effect of $\mathrm{CPAP}$ treatment, it is relevant to evaluate eventual changes in vasoactive hor- 
mones and renal handling of sodium and water as an explanation for BP response. The lack of changes in plasma levels of hormones in the present study may be related treatment with antihypertensive agent blocking the RAAS system.

Strength of this study is that we at follow-up, in addition to adherence registrations, completed sleep examination in all patients to confirm the use of CPAP-treatment on improved sleep quality (AHI, ODI, and oxygen saturation).

The response on central BP was evaluated from $24 \mathrm{~h}$ measurements, and albuminuria was evaluated from $24 \mathrm{~h}$ urine collection instead of spot urine measurement. We included a relatively new plasma marker of cardiovascular stress, syndecan-1.

It is a weakness that time from baseline to follow-up time varied from 72 to 139 day. However, we aimed to evaluate the effect short term treatment and defined that as approximately three to four months of intervention, and we did not expect amplification of findings within the present used timeframe. Another weakness of the study is that a few subjects experienced changes in antihypertensive treatment. However, it was not ethically justified not to treat these patients with usual care, and the change in mean DDD was only minor. We did not include a sham or placebo intervention to eliminate the effect of biases or to address the Hawthorne effect.

\section{Conclusion}

CPAP seems to be an effective blood pressure lowering treatment in patients with mean eGFR $66 \mathrm{~mL} / \mathrm{min} / 1.73 \mathrm{~m}^{2}$ and sleep apnoea with beneficial effects on albuminuria. Larger studies are needed to verify this finding and explore potential effects on the progression of renal failure.

\section{Acknowledgements}

The authors greatly acknowledge the skillful assistance of our laboratory technicians: Anne Mette Ravn, Kirsten Nygaard, and Henriette Vorup Simonsen.

The authors also greatly acknowledge the skilful assistance from Nurses Marianne Kirkegaard and Anja Mailund Mikkelsen in the Sleep Apnoea Clinic, Department of Medicine, Holstebro Hospital.

The authors thank the Department of Clinical Biochemistry, Holstebro Hospital for help in routine analyses.

\section{Conflicts of Interest}

The authors have no conflicts of interest.

\section{Funding}

This research project has received research funding from The Central Denmark Region's Research Foundation for Health Science, the Danish Heart Association, and the Axel Muusfeldt Foundation. 


\section{References}

[1] Nicholl, D.D., Ahmed, S.B., Loewen, A.H., Hemmelgarn, B.R., Sola, D.Y., Beecroft, J.M., Turin, T.C. and Hanly, P.J. (2012) Declining Kidney Function Increases the Prevalence of Sleep Apnea and Nocturnal Hypoxia. Chest, 141, 1422-1430. https://doi.org/10.1378/chest.11-1809

[2] Worsnop, C.J., Naughton, M.T., Barter, C.E., Morgan, T.O., Anderson, A.I. and Pierce, R.J. (1998) The Prevalence of Obstructive Sleep Apnea in Hypertensives. American Journal of Respiratory and Critical Care Medicine, 157, 111-115. https://doi.org/10.1164/ajrccm.157.1.9609063

[3] Yaggi, H.K., Concato, J., Kernan, W.N., Lichtman, J.H., Brass, L.M. and Mohsenin, V. (2005) Obstructive Sleep Apnea as a Risk Factor for Stroke and Death. The New England Journal of Medicine, 353, 2034-2041. https://doi.org/10.1056/NEJMoa043104

[4] Drager, L.F., Bortolotto, L.A., Lorenzi, M.C., Figueiredo, A.C., Krieger, E.M. and Lorenzi-Filho, G. (2005) Early Signs of Atherosclerosis in Obstructive Sleep Apnea. American Journal of Respiratory and Critical Care Medicine, 172, 613-618. https://doi.org/10.1164/rccm.200503-340OC

[5] Sakaguchi, Y., Hatta, T., Hayashi, T., Shoji, T., Suzuki, A., Tomida, K., Okada, N., Rakugi, H., Isaka, Y. and Tsubakihara, Y. (2013) Association of Nocturnal Hypoxemia with Progression of CKD. Clinical Journal of the American Society of Nephrology, 8, 1502-1507. https://doi.org/10.2215/CJN.11931112

[6] Marrone, O., Battaglia, S., Steiropoulos, P., Basoglu, O.K., Kvamme, J.A., Ryan, S., Pepin, J.L., Verbraecken, J., Grote, L., Hedner, J., Bonsignore, M.R. and ESADA Study Group (2016) Chronic Kidney Disease in European Patients with Obstructive Sleep Apnea: The ESADA Cohort Study. Journal of Sleep Research, 25, 739-745. https://doi.org/10.1111/jsr.12426

[7] Giles, T.L., Lasserson, T.J., Smith, B.H., White, J., Wright, J. and Cates, C.J. (2006) Continuous Positive Airways Pressure for Obstructive Sleep Apnoea in Adults. Cochrane Database of Systematic Reviews, 25, CD001106.

[8] Montserrat, J.M., Ferrer, M., Hernandez, L., Farre, R., Vilagut, G., Navajas, D., Badia, J.R., Carrasco, E., De Pablo, J. and Ballester, E. (2001) Effectiveness of CPAP Treatment in Daytime Function in Sleep Apnea Syndrome: A Randomized Controlled Study with an Optimized Placebo. American Journal of Respiratory and Critical Care Medicine, 164, 608-613. https://doi.org/10.1164/ajrccm.164.4.2006034

[9] Sullivan, C.E., Issa, F.G., Berthon-Jones, M. and Eves, L. (1981) Reversal of Obstructive Sleep Apnoea by Continuous Positive Airway Pressure Applied through the Nares. The Lancet, 317, 862-865. https://doi.org/10.1016/S0140-6736(81)92140-1

[10] Puckrin, R., Iqbal, S., Zidulka, A., Vasilevsky, M. and Barre, P. (2015) Renoprotective Effects of Continuous Positive Airway Pressure in Chronic Kidney Disease Patients with Sleep Apnea. International Urology and Nephrology, 47, 1839-1845. https://doi.org/10.1007/s11255-015-1113-y

[11] Schipper, M.H., Jellema, K., Thomassen, B.J.W., Alvarez-Estevez, D., Verbraecken, J. and Rijsman, R.M. (2017) Stroke and Other Cardiovascular Events in Patients with Obstructive Sleep Apnea and the Effect of Continuous Positive Airway Pressure. Journal of Neurology, 264, 1247-1253.

https://doi.org/10.1007/s11255-015-1113-y

[12] Campos-Rodriguez, F., Perez-Ronchel, J., Grilo-Reina, A., Lima-Alvarez, J., Benitez, M.A. and Almeida-Gonzalez, C. (2007) Long-Term Effect of Continuous Positive 
Airway Pressure on BP in Patients with Hypertension and Sleep Apnea. Chest, 132, 1847-1852. https://doi.org/10.1378/chest.07-1478

[13] Campos-Rodriguez, F., Grilo-Reina, A., Perez-Ronchel, J., Merino-Sanchez, M., Gonzalez-Benitez, M.A., Beltran-Robles, M. and Almeida-Gonzalez, C. (2006) Effect of Continuous Positive Airway Pressure on Ambulatory BP in Patients with Sleep Apnea and Hypertension: A Placebo-Controlled Trial. Chest, 129, 1459-1467. https://doi.org/10.1378/chest.129.6.1459

[14] Bazzano, L.A., Khan, Z., Reynolds, K. and He, J. (2007) Effect of Nocturnal Nasal Continuous Positive Airway Pressure on Blood Pressure in Obstructive Sleep Apnea. Hypertension, 50, 417-423. https://doi.org/10.1161/HYPERTENSIONAHA.106.085175

[15] Williams, B., Lacy, P.S., Yan, P., Hwee, C.N., Liang, C. and Ting, C.M. (2011) Development and Validation of a Novel Method to Derive Central Aortic Systolic Pressure from the Radial Pressure Waveform Using an N-Point Moving Average Method. Journal of the American College of Cardiology, 57, 951-961. https://doi.org/10.1016/j.jacc.2010.09.054

[16] Roman, M.J., Devereux, R.B., Kizer, J.R., Lee, E.T., Galloway, J.M., Ali, T., Umans, J.G. and Howard, B.V. (2007) Central Pressure More Strongly Relates to Vascular Disease and Outcome than Does Brachial Pressure: The Strong Heart Study. Hypertension, 50, 197-203. https://doi.org/10.1161/HYPERTENSIONAHA.107.089078

[17] Korcarz, C.E., Benca, R., Barnet, J.H. and Stein, J.H. (2016) Treatment of Obstructive Sleep Apnea in Young and Middle-Aged Adults: Effects of Positive Airway Pressure and Compliance on Arterial Stiffness, Endothelial Function, and Cardiac Hemodynamics. Journal of the American Heart Association, 5, e002930. https://doi.org/10.1161/JAHA.115.002930

[18] Tarbell, J.M. and Cancel, L.M. (2016) The Glycocalyx and Its Significance in Human Medicine. Journal of Internal Medicine, 280, 97-113. https://doi.org/10.1111/joim.12465

[19] Hornstrup, B.G. (2018) Nocturnal Blood Pressure Decrease in Hypertensive Patients and Normotensives-Association with Obstructive Sleep Apnoea and Renal Function. The Open Hypertension Journal, 10, 28-40. https://doi.org/10.2174/1876526201810010028

[20] Hoffmann-Petersen, N., Lauritzen, T., Bech, J.N. and Pedersen, E.B. (2017) Short-Term Telemedical Home Blood Pressure Monitoring Does Not Improve Blood Pressure in Uncomplicated Hypertensive Patients. Journal of Human Hypertension, 31, 93-98. https://doi.org/10.1038/jhh.2016.43

[21] Hornstrup, B.G., Gjoerup, P.H., Wessels, J., Lauridsen, T.G. and Pedersen, E.B. (2018) Nocturnal Blood Pressure Decrease in Patients with Chronic Kidney Disease and in Healthy Controls-Significance of Obstructive Sleep Apnea and Renal Function. International Journal of Nephrology and Renovascular Disease, 11, 279-290. https://doi.org/10.2147/IJNRD.S176606

[22] ESH/ESC Task Force for the Management of Arterial Hypertension (2013) Practice Guidelines for the Management of Arterial Hypertension of the European Society of Hypertension (ESH) and the European Society of Cardiology (ESC): ESH/ESC Task Force for the Management of Arterial Hypertension. Journal of Hypertension, 31, 1925-1938. https://doi.org/10.1097/HJH.0b013e328364ca4c

[23] Berry, R.B., Budhiraja, R., Gottlieb, D.J., Gozal, D., Iber, C., Kapur, V.K., Marcus, C.L., Mehra, R., Parthasarathy, S., Quan, S.F., Redline, S., Strohl, K.P., Davidson 
Ward, S.L. and Tangredi, M.M. (2012) American Academy of Sleep Medicine: Rules for Scoring Respiratory Events in Sleep: Update of the 2007 AASM Manual for the Scoring of Sleep and Associated Events. Deliberations of the Sleep Apnea Definitions Task Force of the American Academy of Sleep Medicine. Journal of Clinical Sleep Medicine, 8, 597-619.

[24] Pedersen, E.B., Eiskjaer, H., Madsen, B., Danielsen, H., Egeblad, M. and Nielsen, C.B. (1993) Effect of Captopril on Renal Extraction of Renin, Angiotensin II, Atrial Natriuretic Peptide and Vasopressin, and Renal Vein Renin Ratio in Patients with Arterial Hypertension and Unilateral Renal Artery Disease. Nephrology Dialysis Transplantation, 8, 1064-1070.

[25] Pedersen, E.B., Danielsen, H. and Spencer, E.S. (1984) Effect of Indapamide on Renal Plasma Flow, Glomerular Filtration Rate and Arginine Vasopressin in Plasma in Essential Hypertension. European Journal of Clinical Pharmacology, 26, 543-547. https://doi.org/10.1007/BF00543482

[26] Pedersen, R.S., Bentzen, H., Bech, J.N. and Pedersen, E.B. (2001) Effect of Water Deprivation and Hypertonic Saline Infusion on Urinary AQP2 Excretion in Healthy Humans. American Journal of Physiology-Renal Physiology, 280, F860-F867. https://doi.org/10.1152/ajprenal.2001.280.5.F860

[27] Graffe, C.C., Bech, J.N. and Pedersen, E.B. (2012) Effect of High and Low Sodium Intake on Urinary Aquaporin-2 Excretion in Healthy Humans. American Journal of Physiology-Renal Physiology, 302, F264-F275. https://doi.org/10.1152/ajprenal.00442.2010

[28] Matthesen, S.K., Larsen, T., Vase, H., Lauridsen, T.G., Jensen, J.M. and Pedersen, E.B. (2013) Effect of Amiloride and Spironolactone on Renal Tubular Function and Central Blood Pressure in Patients with Arterial Hypertension during Baseline Conditions and after Furosemide: A Double-Blinded, Randomized, Placebo-Controlled Crossover Trial. Clinical and Experimental Hypertension, 35, 313-324. https://doi.org/10.3109/10641963.2012.721843

[29] Al Therwani, S., Malmberg, M.E.S., Rosenbaek, J.B., Bech, J.N. and Pedersen, E.B. (2017) Effect of Tolvaptan on Renal Handling of Water and Sodium, GFR and Central Hemodynamics in Autosomal Dominant Polycystic Kidney Disease during Inhibition of the Nitric Oxide System: A Randomized, Placebo-Controlled, Double Blind, Crossover Study. BMC Nephrology, 18, 268.

[30] Hays, R.D., Sherbourne, C.D. and Mazel, R.M. (1993) The RAND 36-Item Health Survey 1.0. Health Economics, 2, 217-227. https://doi.org/10.1002/hec.4730020305

[31] Becker, H.F., Jerrentrup, A., Ploch, T., Grote, L., Penzel, T., Sullivan, C.E. and Peter, J.H. (2003) Effect of Nasal Continuous Positive Airway Pressure Treatment on Blood Pressure in Patients with Obstructive Sleep Apnea. Circulation, 107, 68-73. https://doi.org/10.1161/01.CIR.0000042706.47107.7A

[32] Hermida, R.C., Zamarron, C., Ayala, D.E. and Calvo, C. (2004) Effect of Continuous Positive Airway Pressure on Ambulatory Blood Pressure in Patients with Obstructive Sleep Apnoea. Blood Pressure Monitoring, 9, 193-202.

https://doi.org/10.1097/00126097-200408000-00004

[33] Duran-Cantolla, J., Aizpuru, F., Montserrat, J.M., Ballester, E., Teran-Santos, J., Aguirregomoscorta, J.I., Gonzalez, M., Lloberes, P., Masa, J.F., De La Pena, M., Carrizo, S., Mayos, M., Barbe, F., Spanish Sleep and Breathing Group (2010) Continuous Positive Airway Pressure as Treatment for Systemic Hypertension in People with Obstructive Sleep Apnoea: Randomised Controlled Trial. BMJ, 341, c5991. https://doi.org/10.1136/bmj.c5991 
[34] Litvin, A.Y., Sukmarova, Z.N., Elfimova, E.M., Aksenova, A.V., Galitsin, P.V., Rogoza, A.N. and Chazova, I.E. (2013) Effects of CPAP on "Vascular" Risk Factors in Patients with Obstructive Sleep Apnea and Arterial Hypertension. Vascular Health and Risk Management, 9, 229-235. https://doi.org/10.2147/VHRM.S40231

[35] Hoyos, C.M., Yee, B.J., Wong, K.K., Grunstein, R.R. and Phillips, C.L. (2015) Treatment of Sleep Apnea with CPAP Lowers Central and Peripheral Blood Pressure Independent of the Time-of-Day: A Randomized Controlled Study. American Journal of Hypertension, 28, 1222-1228. https://doi.org/10.1093/ajh/hpv023

[36] Koga, S., Ikeda, S., Yasunaga, T., Nakata, T. and Maemura, K. (2013) Effects of Nasal Continuous Positive Airway Pressure on the Glomerular Filtration Rate in Patients with Obstructive Sleep Apnea Syndrome. Internal Medicine, 52, 345-349. https://doi.org/10.2169/internalmedicine.52.8468

[37] Barbe, F., Duran-Cantolla, J., Capote, F., de la Pena, M., Chiner, E., Masa, J.F., Gonzalez, M., Marin, J.M., Garcia-Rio, F., de Atauri, J.D., Teran, J., Mayos, M., Monasterio, C., del Campo, F., Gomez, S., de la Torre, M.S., Martinez, M., Montserrat, J.M., Spanish Sleep and Breathing Group (2010) Long-Term Effect of Continuous Positive Airway Pressure in Hypertensive Patients with Sleep Apnea. American Journal of Respiratory and Critical Care Medicine, 181, 718-726. https://doi.org/10.1164/rccm.200901-0050OC

[38] Zhang, J., Wang, C., Gong, W., Ye, Z., Tang, Y., Zhao, W., Peng, H. and Lou, T. (2016) Poor Sleep Quality Is Responsible for the Non-Dipper Pattern in Hypertensive But Not in Normotensive Chronic Kidney Disease Patients. Nephrology (Carlton), 22, 690-698.

[39] Jenkinson, C., Davies, R.J., Mullins, R. and Stradling, J.R. (1999) Comparison of Therapeutic and Subtherapeutic Nasal Continuous Positive Airway Pressure for Obstructive Sleep Apnoea: A Randomised Prospective Parallel Trial. The Lancet, 353, 2100-2105. https://doi.org/10.1016/S0140-6736(98)10532-9

[40] Padberg, J.S., Wiesinger, A., di Marco, G.S., Reuter, S., Grabner, A., Kentrup, D., Lukasz, A., Oberleithner, H., Pavenstadt, H., Brand, M. and Kumpers, P. (2014) Damage of the Endothelial Glycocalyx in Chronic Kidney Disease. Atherosclerosis, 234, 335-343. https://doi.org/10.1016/j.atherosclerosis.2014.03.016

[41] Bielecka-Dabrowa, A., Michalska-Kasiczak, M., Gluba, A., Ahmed, A., Gerdts, E., von Haehling, S., Rysz, J. and Banach, M. (2015) Biomarkers and Echocardiographic Predictors of Myocardial Dysfunction in Patients with Hypertension. Scientific Reports, 5, Article No. 8916. https://doi.org/10.1038/srep08916

[42] Gaddam, K., Pimenta, E., Thomas, S.J., Cofield, S.S., Oparil, S., Harding, S.M. and Calhoun, D.A. (2010) Spironolactone Reduces Severity of Obstructive Sleep Apnoea in Patients with Resistant Hypertension: A Preliminary Report. Journal of Human Hypertension, 24, 532-537. https://doi.org/10.1038/jhh.2009.96

[43] Follenius, M., Krieger, J., Krauth, M.O., Sforza, F. and Brandenberger, G. (1991) Obstructive Sleep Apnea Treatment: Peripheral and Central Effects on Plasma Renin Activity and Aldosterone. Sleep, 14, 211-217.

[44] Yang, L., Zhang, H., Cai, M., Zou, Y., Jiang, X., Song, L., Liang, E., Bian, J., Wu, H. and Hui, R. (2016) Effect of Spironolactone on Patients with Resistant Hypertension and Obstructive Sleep Apnea. Clinical and Experimental Hypertension, 38, 464-468. https://doi.org/10.3109/10641963.2015.1131290 